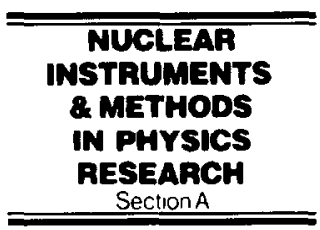

\title{
Construction and calibration of detectors for high-resolution metabolic breast cancer imaging
}

\author{
J.L. Robar*, C.J. Thompson, K. Murthy, R. Clancy, A.M. Bergman \\ Montreal Neurological Institute, Room 798, Research Computing Lab, 3801 University Street, Montreal, Quebec, Canada H3A $2 B 4$
}

\begin{abstract}
Each of two detectors used in our Positron Emission Mammography (PEM) system consists of four $36 \mathrm{~mm} \times$ $36 \mathrm{~mm} \times 20 \mathrm{~mm}$ bismuth germanate (BGO) crystal detector blocks coupled to a crossed-wire anode position-sensitive photomultiplier tube (PS-PMT). To facilitate high spatial-resolution imaging, the crystal blocks have been finely pixelated using a diamond saw. In each detector, $36 \times 361.9 \mathrm{~mm} \times 1.9 \mathrm{~mm}$ crystal elements are coupled directly to the PMT window and, on the opposite face of the blocks, $35 \times 35$ elements are offset by $1.0 \mathrm{~mm}$ along both the $x$ - and $y$-axis of the PS-PMT. As part of a system calibration routine, a novel method for crystal element identification has been developed. This algorithm successfully identifies $59 \times 49$ crystal elements on each detector face. These results are used to generate a Look-Up-Table (LUT) that is accessed during image formation for the effective correction of spatial distortion inherent in the detectors. Crystal identification also facilitates the capability for accurate energy discrimination, since the detector gain is considered on an element-by-element basis by accessing an energy LUT. Employing a third LUT, which contains the relative efficiencies of individual crystal elements results in improvement in image uniformity from $50 \%$ to $13 \%$.
\end{abstract}

\section{Introduction}

Breast carcinoma is the second most common malignancy among women in the United States and has an estimated annual mortality rate of 45000 [1]. Mortality is reduced by detecting the cancer at an early stage, because the probability for metastasis increases with tumour volume [2]. Unfortunately, the current X-ray mammographic methods are limited in terms of both sensitivity and specificity. For example, mammographic techniques may fail in the detection of one-third of primary breast cancers [3]. Because mammograms do not provide metabolic or histologic information, any observed suspicious lesion is typically examined using biopsy. Both needle and open biopsies are expensive and traumatic procedures and frequentl. leave scars which interfere with follow-up monitoring ased on subsequent X-ray images. Moreover, $53-91 \%$ of biopsies reveal that the procedure has been performed on a benign tumour [4-6].

The development of detectors for Positron Emission Mammography (PEM) [7,8] has been motivated by

\footnotetext{
*Corresponding author. Tel:: + 15143988506 ; fax: + 1514 398 8106; e-mail: james@rclvax.medcor.mcgill.ca.
}

the results of studies evaluating the role of whole-body Positron Emission Tomography (PET) in breast cancer imaging. The sensitivity of PET for the detection of tumours of diameter $>1.0 \mathrm{~cm}$ has shown to be close to $100 \%$ [1]. Based on a consistent overconsumption of the metabolic tracer F-18-2-deoxy-fluoro-D-glucose (FDG) by malignant lesions compared to benign lesions, FDG PET promises a specificity of over $90 \%$ [1]. Due to its insufficient spatial resolution (typically $4.5-5.5 \mathrm{~mm}$ ), however, the effectiveness of whole-body PET in imaging smaller tumours has not been defined [9]. Other principal shortcomings of PET are its considerable capital cost and limited availability.

We have constructed an affordable high-resolution imaging system that exploits the specificity of FDG PET, but offers improved spatial resolution and approximately 20 times greater efficiency [8]. The capability for high-spatial resolution $(\sim 2.0 \mathrm{~mm})$ imaging is achieved by using finely pixelated bismuth germanate (BGO) modular detector blocks coupled to Hamamatsu R3941-05 position-sensitive photomultipliers (PSPMTs). Modular scintillator blocks have been used widely in PET scanners, and current PET detector blocks are segmented into, for example, a matrix of $8 \times 8 \quad 4.39 \mathrm{~mm} \times 4.05 \mathrm{~mm}$ crystal elements [10]. In comparison, the PEM detector block is segmented on 
one $36 \mathrm{~mm} \times 36 \mathrm{~mm}$ face into $18 \times 181.9 \mathrm{~mm} \times 1.9 \mathrm{~mm}$ elements and into $17 \times 17$ clements on the oppositc side. While this dual-layer design significantly increases the crystal-element packing fraction, it introduces several manufacturing challenges because $\mathrm{BGO}$ is extremely hard and brittle. In addition, we have found that the high-resolution detector block/PS-PMT combination necessitates novel techniques for the correction of three detector characteristics in particular: (i) spatial distortion; (ii) a spatial variation of detector gain; and (iii) a spatial nonuniformity of detector efficiency.

\section{Detector design and manufacture}

A machined PEM block is shown in Fig. 1. The block has dimensions $36 \mathrm{~mm} \times 36 \mathrm{~mm} \times 20 \mathrm{~mm}$, and is segmented into $1.9 \mathrm{~mm} \times 1.9 \mathrm{~mm}$ elements to prevent the spreading of scintillation light through the crystal. The $18 \times 18$ crystal elements cut into the face that is coupled to the PS-PMT (referred to as "proximal" elements) are $11.5 \mathrm{~mm}$ in depth. The $17 \times 17$ "distal" elements on the opposite face are $6.5 \mathrm{~mm}$ in depth. These depths are based on Monte Carlo simulation results to ensure an equal probability of gamma-ray interaction in each of the two layers [8]. All elements remain attached to the modular block by a $2.0 \mathrm{~mm}$ thick uncut region. The distal elements are offset from the proximal elements by $1.0 \mathrm{~mm}$ along both the $x$ - and $y$-axis of the PS-PMT. This arrangement produces an element centre-to-centre separation of $1.4 \mathrm{~mm}$ on the diagonal. The total block thickness of $20 \mathrm{~mm}$ has been selected to achieve a reasonable detector efficiency. One bit of depth-of-interaction in- formation is obtained by identifying the layer in which events occur.

The solid BGO blocks (Alpha Spectra, Grand Junction, $\mathrm{CO}$ ) are cut using a precision saw (Ultra Tec, Santa Ana, CA) with a tolerance of $\pm 0.1 \mathrm{~mm}$. Three diamond-coated, $0.25 \mathrm{~mm}$ thick blades are used simultaneously, separated by $1.85 \mathrm{~mm}$ thick spacers. During the cutting, the $2.0 \mathrm{~mm}$ uncut thickness of the block is secured using a dedicated vice. To increase light output, the coarse surface produced during cutting is subsequently polished by etching the crystal in an ultrasonic bath of nitric and hydrochloric acid. The final step of the manufacturing process involves potting the block by filling the cuts with an epoxy-based, white, opaque material. This serves three purposes by (i) optically isolating adjacent crystal elements; (ii) reflecting photons which have escaped the volume of a crystal element; and (iii) significantly increasing the mechanical strength of the block.

Four blocks are optically coupled to the $73 \mathrm{~mm} \times$ 73 mm Hamamatsu R3941-05 PS-PMT window, resulting in 5041 crystal elements in total per detector. The PS-PMT employs a crossed-wire anode, with 18 wires in the $x$-direction (parallel to the patient's chest) and 16 wires in the $y$-direction, placed at pitches of 3.75 and $3.70 \mathrm{~mm}$, respectively. The wires are connected in an Anger-type resistor chain readout which provides $x^{+}$, $x^{-}, y^{+}, y^{-}$positioning signals.

\section{Detector calibration}

Fast timing signals from the last dynodes of the PSPMTs are used to generate ADC strobe signals. Digitized

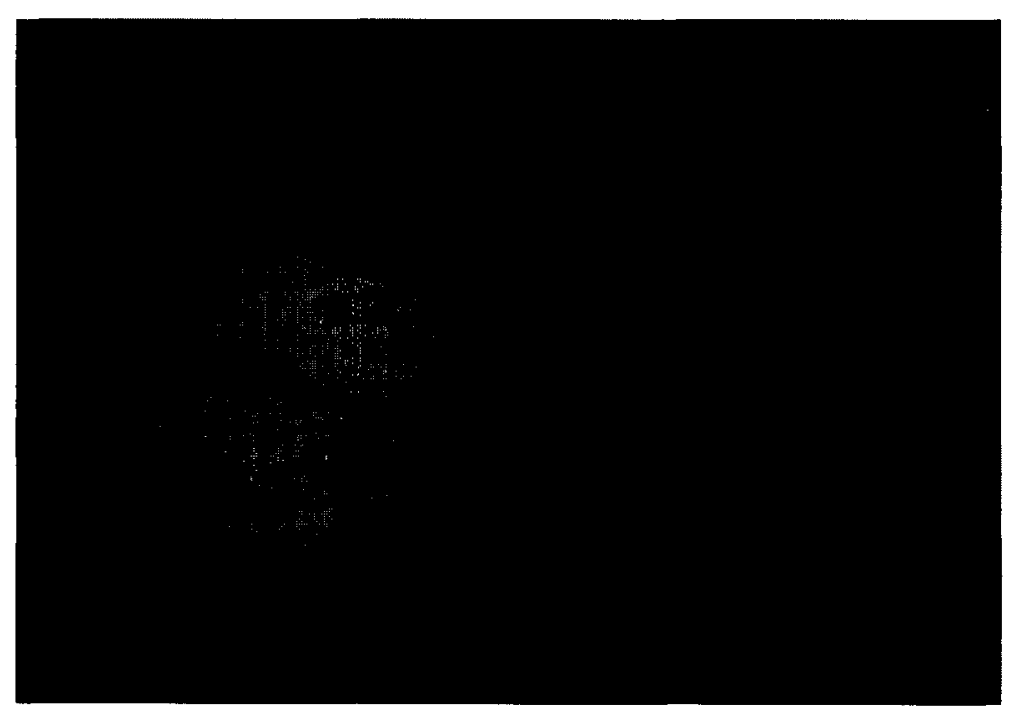

Fig. 1. A PEM BGO detector block. 
$X$ - and $Y$-positioning signals are formed by computing $X=\left(x^{+}-x^{-}\right) / E$ and $Y=\left(y^{+}-y^{-}\right) / E$, respectively, where $E$ is the event energy equal to $\left(x^{+}+x^{-}+y^{+}+y^{-}\right)$. For each detected coincident event, the computed $X, Y$, and $E$ values for each detector are stored sequentially in a list file as 8-bit numbers.

Data for the quantification of spatial distortion is collected by acquiring $3 \times 10^{6}$ singles events while collimating a $5 \mu \mathrm{Ci}{ }^{68} \mathrm{Ge}-{ }^{68} \mathrm{Ga}$ source to irradiate only the proximal crystal elements from the side of the block. Plotting a two-dimensional histogram of $(X, Y)$ pairs produces an image in which distributions from individual elements are clearly separated (with a mean peak-tovalley ratio of 3.8 ), and the effect of the spatial distortion is readily observed. Using a graphical interface written in $C$ and MATLAB for PEM crystal identification, several points between each of the adjacent crystal rows and columns are located manually in this image. These points are then used to computc splinc-interpolated curves which accurately delineate the curvature of the "valleys" in the image. The intersection points of vertical and horizontal spline curves are computed and are allowed to "wander" so that they are shifted to the locations of minima between each $2 \times 2$ group of crystal elements. A $3 \times 3$ smoothing kernel is used in this step to prevent the intersection points from being relocated to minima resulting from image noise. The locations determined in this fashion arc used to specify four-sided polygons around the images of each element, as shown in Fig. 2. This algorithm thus also allocates regions corresponding to the distal crystal elements between each $2 \times 2$ group of proximal elements. In total, $59 \times 49$ crystal element regions have been identified using this method. The locations of boundary corners have been observed to be stationary between successive crystal identifications, with an average standard deviation in position of $0.1 \mathrm{~mm}$. The crystal identification results for each detector are stored in a $256 \times 256$ distortion Look-Up-Table (LUT). This is accessed during image formation to map each $(X, Y)$ pair to the corresponding crystal element coordinates, $(C x$, $C y)$, while correcting for spatial distortion.

The second detector characteristic requiring correction is a marked spatial variation of detector gain. If left uncorrected, this effect precludes performing event energy discrimination using absolute units of energy $(\mathrm{keV})$. To correct for this variation, $3 \times 10^{6}$ singles events are acquired while flood irradiating a detector with a ${ }^{68} \mathrm{Ge}-{ }^{68} \mathrm{Ga}$ source and individual energy spectra are compiled for each of the previously identified crystal elements. An automated routine then locates the photopeak in each spectrum, and stores the element-specific $\mathrm{keV} / \mathrm{ADC}$ conversion factors in an energy LUT.

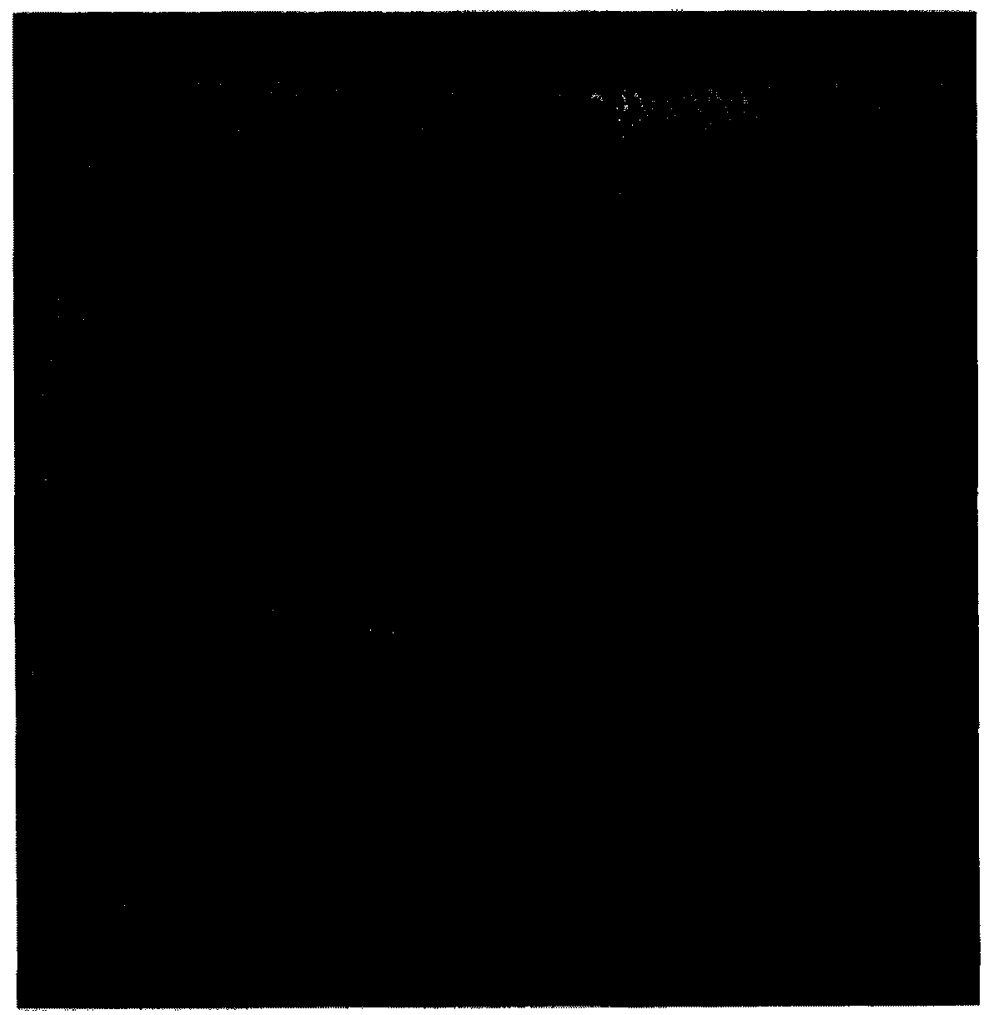

Fig. 2. The crystal identification image with the crystal element boundaries superimposed. 
The same flood-irradiation list file is used to record the relative number of events detected by each of the identified elements. This information is written to a $72 \times 72$ LUT and is accessed during data acquisition to compensate for the spatial nonuniformity of detector efficiency.

\section{Results}

\subsection{Detector construction}

The techniques described for crystal manufacture have resulted in a successful yield, with $\sim 99 \%$ of the small crystal elements remaining intact throughout the cutting, etching and potting stages. Approximately one week is

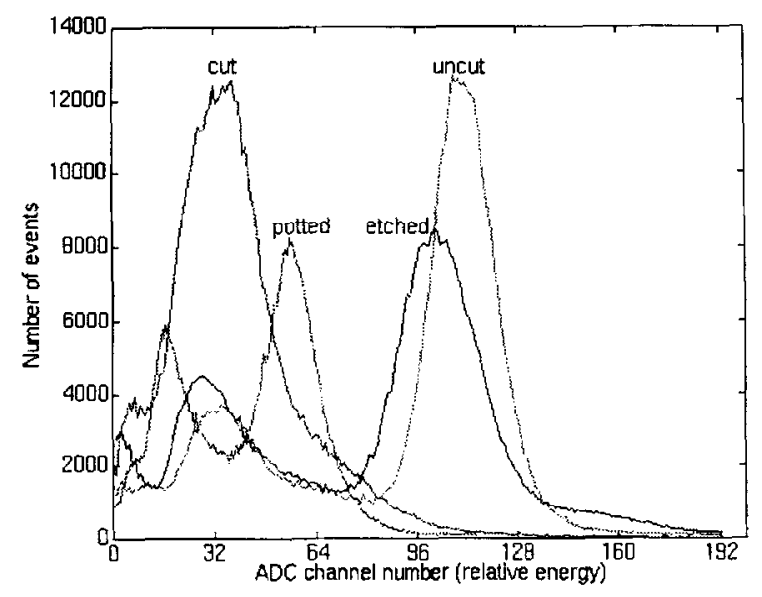

Fig. 3. The scintillation light output varies according to the stage of crystal block manufacture as indicated by the relative locations of energy spectra photopeaks. required to machine the eight crystals required to complete a full PEM system.

The light output and energy resolution were monitored at each stage of manufacture by coupling a single detector block to the centre of the PS-PMT window and acquiring singles data while flood-irradiating the detector. Typical spectra for the same block are shown in Fig. 3. The crystal cutting degrades the light output drastically, as indicated by a shift in the spectrum photopeak from channel 109 to 37 . The acid-etching recovers almost all of this light, increasing the photopeak channel to 102. Crystal potting reduces the light output because the epoxy-based filler makes optical contact with the BGO, increasing the critical angle for total internal reflection. The average final energy resolution for a single potted block is $53 \%$, but the effective value is improved significantly by using an energy LUT as explained below.

In order to examine the dependence of the spatial resolution on the phase of manufacturing, single PEM blocks were coupled to the centres of each of two PS-PMTs separated by $10 \mathrm{~cm}$, and were irradiated by ${ }^{68} \mathrm{Ge}-{ }^{68} \mathrm{Ga}$ point sources at the midpoint of the two detectors. Coincident events were acquired and backprojected as described

Table 1

The full-width-half-maximum (FWHM) of the coincident image of a point source decreases at each stage of detector block manufacture

\begin{tabular}{ll}
\hline Stage of manufacture & FWHM (mm) \\
\hline Uncut & Not resolved \\
Cut & 3.22 \\
Cut and etched & 2.61 \\
Cut, etched and potted & 2.05 \\
\hline
\end{tabular}

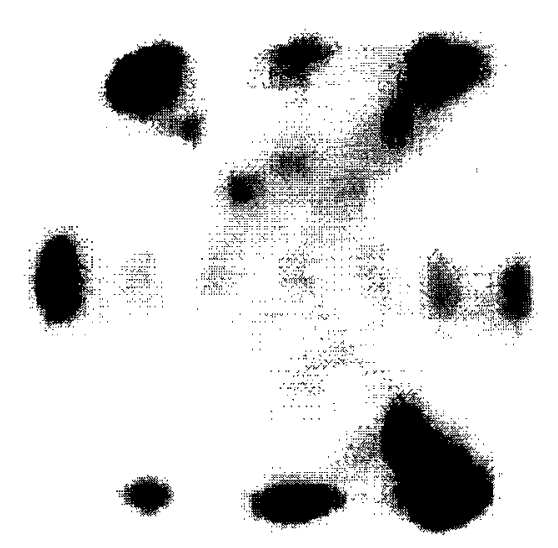

a)

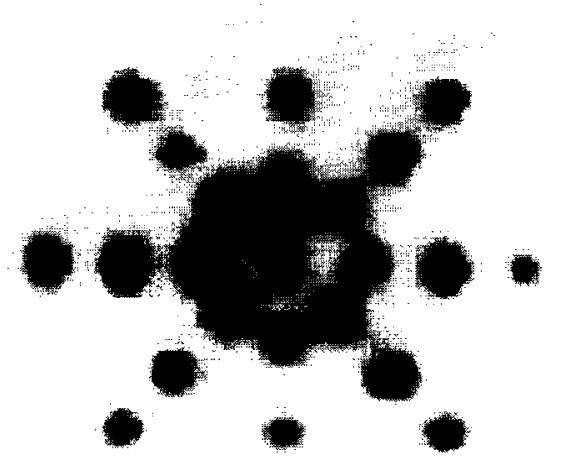

b)

Fig. 4. The image of $231 \mathrm{~mm}$ diameter capillary tubes oriented perpendicular to the detector faces and filled with FDG (a) without spatial distortion and efficiency corrections; and (b) with the application of distortion and efficiency LUTs. 
in Ref. [8], using a 300-700 keV energy window. As listed in Table 1, the measured FWIIM of the point-source distributions decreases progressively with each stage of manufacture. The FWHM for the final potted block is $2.05 \mathrm{~mm}$.

\subsection{Detector calibration}

Fig. 4 illustrates the effects of the distortion and efficiency LUTs on an image of an arrangement of $1 \mathrm{~mm}$ diameter capillary tubes placed perpendicular to the detector faces and filled with $2.5 \mathrm{mCi} / \mathrm{ml}$ FDG. The distortion LUT clearly corrects both the shape and relative locations of the images of each tube. The efficiency LUT compensates for the disproportionate number of counts occurring in the most peripheral tubes of Fig. 4(a).

The effect of applying the efficiency LUT on the coincident image was examined quantitatively by acquiring a coincident image of a flood source consisting of a $2 \mu \mathrm{Ci} / \mathrm{ml} \mathrm{FDG} \mathrm{solution} \mathrm{in} \mathrm{a} 80 \mathrm{~mm} \times 80 \mathrm{~mm} \times 40 \mathrm{~mm}$ container. The nonuniformity was described by $(\max -\min ) /(\max +\min ) \times 100 \%$ (where $\max$ and $\min$ are the maximum and minimum image pixel values), and

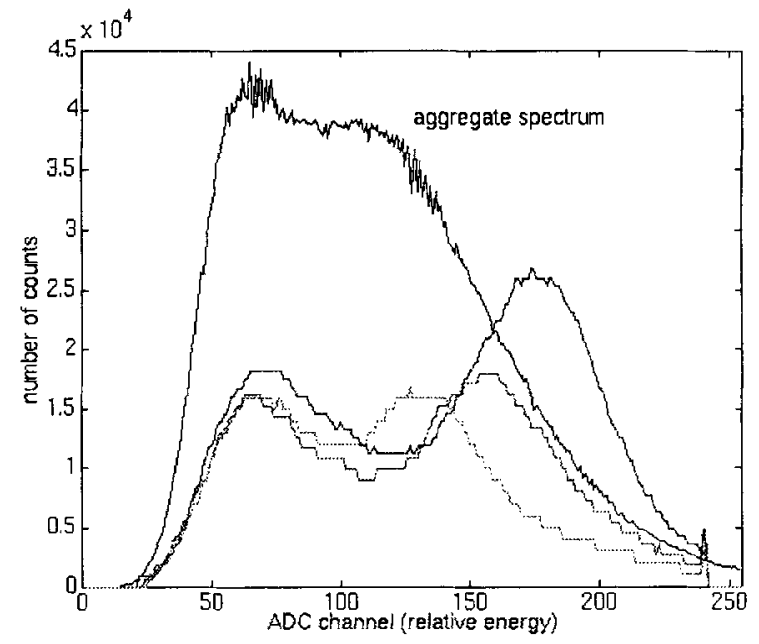

Fig. 5. The aggregate singles energy spectrum for four detector blocks, and the spectra for three identified crystal elements (element spectra have been scaled for plotting on the aggregate spectrum scale). The average single-element energy resolution is $35 \%$. was reduced from $50 \%$ to $13 \%$ by applying the efficiency LUT.

Fig. 5 illustrates that the aggregate energy spectrum for four potted blocks precludes performing energy discrimination entirely, due to the wide variation of both photocathode quantum efficiency [11] and crystal element light output over the detector area. The photopeak is recovered, however, when an energy spectrum is compiled for a single identified element. Energy discrimination is therefore performed on an element-by-element basis using the energy LUT. The average single-element energy resolution is $35 \%$.

\section{Conclusion}

The novel PEM detector described here is feasible in terms of its manufacture, and with the application of appropriate corrections for spatial distortion, spatial variation of gain, and efficiency nonuniformity, is capable of accurate high-resolution metabolic imaging. The calibration routines described here may be completed over several hours, and are sufficiently straightforward to be implemented in a clinical setting. The detectors are currently in use during the initial clinical trials of the PEM scanner at the Royal Victoria Hospital in Montreal.

\section{References}

[1] L.P. Adler, J.P. Crowe, N.K. Al-Kaisi and J.L. Sunshine, Radiology 187 (1993) 743.

[2] D.E. Henson and L.A. Ries, Cancer 65 (1990) 2155.

[3] J. Gisvold, Mayo Clin. Proc. 65 (1990) 56.

[4] N. Lang, N. Talbert and K. Shewmake, Arch. Surg. 122 (1987) 1389.

[5] M. Skinner, M. Swain and R. Simmons, Ann. Surg. 208 (1988) 203.

[6] D. Franceschi, J. Crowe, R. Zollinger et al., Arch. Surg. 125 (1990) 170.

[7] C.J. Thompson, K. Murthy, I.N. Weinberg and R. Mako, Med. Phys. 21 (1994) 529.

[8] C.J. Thompson, K. Murthy, Y. Picard, 1.N. Weinberg and R. Mako, IEEE Trans. Nucl. Sci. NS-42 (1995) 1012.

[9] D.D. Adler and R.L. Wahl, AYR 164 (1995) 19.

[10] S.R. Cherry, M.P. Tornai, C.S. Levin, S. Siegel and E.J. Hoffman, IEEE Trans. Nucl. Sci. NS-42 (1995) 1064.

「117 N.J. Yasillo, R.N. Beck and M. Cooper, IEEE Trans. Nucl. Sci. NS-37 (1990) 609. 